\title{
Combined evaluation of long-lasting insecticidal nets, house screening and push-pull technology as appropriate interventions for integrated management of malaria vectors and cereal pests in Ethiopia: study protocol for household randomized controlled trial
}

Abebe Asale ( $\square$ aasale@icipe.org )

International Centre of Insect Physiology and Ecology

Menale Kassie

International Centre of Insect Physiology and Ecology

Zewdu Abro

International Centre of Insect Physiology and Ecology

Bayu Enchalew

International Centre of Insect Physiology and Ecology

Aklilu Belay

University of Pretoria, Institute for Sustainable Malaria Control, School of Health Systems and Public Health

\section{Peter 0 Sangoro}

International Centre of Insect Physiology and Ecology

David Tchouassi

International Centre of Insect Physiology and Ecology

Clifford M Mutero

University of Pretoria, Institute for Sustainable Malaria Control, School of Health Systems and Public Health

\section{Research Article}

Keywords: Malaria, study Protocol, RCT, Housing, House screening, Long-lasting insecticidal nets, Vector control, Jabi Tehnan, Ethiopia

Posted Date: May 26th, 2021

DOl: https://doi.org/10.21203/rs.3.rs-558964/v1 
License: (c) (i) This work is licensed under a Creative Commons Attribution 4.0 International License. Read Full License 


\section{Abstract}

Introduction: The combined application of long-lasting insecticidal nets (LLINs) and indoor residual spraying (IRS) has led to mixed results (Significant reduction vs no impact) on malaria burden. Other studies have attempted to study the combined effects of house screening (HS) and LLINs have demonstrated a reduction in indoor vector densities and malaria when the interventions are combined. In addition to malaria vectored by mosquitoes, farmers living in rural settings face challenges from crop pests, which significantly reduce agricultural productivity and negatively impact their food security. Thus, addressing the coexisting risks of malaria and crop pests could contribute to improved livelihood of communities.

Design: A three armed household, cluster-randomized, controlled study will be conducted to assess the impact of HS, LLINs and PPT against clinical malaria in children than LLINs alone in Ethiopia. The unit of randomization will be the household, which include a house and its occupants. A total of 663 households will be enrolled in this study. All the households will receive the LLINs. Then other treatments will be overlaid according to the randomization sequence. Thus, 246 households will receive HS, and 250 households will receive HS and PPT before the commencement of clinical follow-up. The rest 167 houses which received LLINs only will be used as control. One child aged not more than 14 years will be enrolled from each household and followed for clinical malaria using active case detection to estimate malaria incidence for two malaria transmission seasons.

Results: Episodes of clinical malaria, the prevalence of anaemia, density of malaria vector mosquitoes, sporozoite infection rate, crop infestation rate due to stem borer and fall army worm, crop yield gain, livestock productivity and cost effectiveness analysis will be the end points of this study. Socioeconomic, social demographic, cost-effectiveness analysis will be conducted using qualitative and participatory methods to explore the acceptability of HS and PPT.

Conclusion Documenting the combined impact of LLINs, HS and PPT on the prevalence of clinical malaria and crop pest damage will be the first of its kind and will provide deep insight and evidence base for forging collaborations between health and agriculture sectors.

Trial Registration: The protocol was registered online on Date 28/05/2020 on sight www.pactr.org With registration No: PACTR202006878245287.

\section{Introduction}

\subsection{Background}

Malaria continues to be a major health threat in Africa where $93 \%$ of the global cases and $94 \%$ of deaths are reported[1]. Long-lasting insecticidal nets (LLINs), indoor residual spraying (IRS), rapid diagnostic tests (RDTs) and prompt disease treatment are key strategies being used in fighting the disease [2]. Despite the proven history of effectiveness in malaria control, both LLINs and IRS interventions are 
insecticide-based, and their effectiveness maybe undermined by widespread occurrence of insecticide resistance in vector populations [3, 4]. Thus, the WHO Global technical strategy emphasises the need to expand the search for innovative vector control strategies or approaches to accelerate malaria elimination efforts [2].

House screening (HS), one of promising supplementary vector control tools, is a valuable non-chemical strategy for preventing indoor biting by vectors, by acting as a physical barrier against entry into human dwellings [5-8]. HS could serve as a potential alternative to IRS, thereby reducing the dependence on chemical insecticides for malaria control. Recent studies have demonstrated that house screening can significantly reduce the number of mosquitoes entering houses in the Gambia [9, 10], Tanzania [11] and Ethiopia $[12,13]$. Despite this evidence, more studies are required to quantify this effect on a larger scale and in different eco-epidemiological strata. A large trial was being implemented in the Gambia to evaluate if improved house screening has an impact on clinical episodes of malaria, the benchmark measurement for assessing malaria interventions and the results just communicated in recent report showing not much expected differences $[14,15]$. We propose to conduct a similar study in Ethiopia to increase the evidence base for this intervention.

The combined application of LLINs and IRS has led to mixed results, with some studies reporting significant reduction in malaria incidence [16,17], and others not finding any difference due to combining the two interventions as compared to using either, alone $[18,19]$. Such studies on combined interventions are generally very few. Thus, in this study we propose to investigate the combined effect of LLINs and HS on entomological parameters such as indoor vector mosquito density, indoor-outdoor resting and indoor-outdoor feeding behaviour, Plasmodium infection rates in vector mosquitoes, and other epidemiological parameters such as malaria prevalence and incidence in the human population.

On the other hand, agriculture and health are interconnected in many ways. Increasing agricultural productivity improves the overall livelihoods of communities through improved health, nutrition, and income generation through sale of crops and livestock [20]. A study conducted in Uganda suggests that a $10 \%$ increase in overall household income would reduce malaria incidence by $35.6 \%$ and, consequently, increase agricultural productivity [21]. In this study, in addition to the combined effect of LLINs and HS on malaria, the integration of push-pull technology (PPT) designed to control cereal stemborers will also be investigated for its impact on farmers agricultural productivity. The benefits of HS and PPT have been separately documented by several studies [8;22-27]. Despite the evidence on the benefits, adoption of either intervention is quite limited. While PPT is adopted by only 158,000 farmers in sub-Saharan Africa [28], HS is still at the experimental stage [14; 29-30]. The low adoption of these technologies necessitates not only introducing them in places where they are needed, but also evaluating their impact on health and livelihoods of the adopters of the technologies. How we best promote the technologies in the current study is also particularly interesting and will help draw lessons for the rest of Africa where the agricultural and health extension systems follow mostly a top-down approach of reaching farmers rather than being participatory [31]. 


\section{Trial Objectives}

\section{General objective}

The general objective of this intervention study is to determine whether the combined intervention of longlasting insecticidal nets, house screening and push-pull technology provides additional protection against malaria and improves agricultural productivity in the intervention area area compared to a control area where only LLINs are in use.

\section{Specific objectives}

i. To determine whether adding screening windows and doors of houses and implementing PPT?? reduces the rate of malaria parasite infection, parasite density, and anemia in children compared to situations where only LLINs are used;

ii. To assess whether the interventions proposed reduce human mosquito-interaction (human biting rates, mosquito resting density, longevity, sporozoite rates, and the entomological inoculation rate (EIR)) inside houses compared with LLINs alone;

iii. To determine the incremental costs, benefits, and cost-effectiveness of adding house screening and PPT to usage of LLINs;

iv.

v. To determine the economic, social, and environmental feasibility of the combined intervention of house screening, LLINs and PPT.

\section{Methods And Materials}

\subsection{Study setting and period}

This study will take place between September 2020 and December 2022 in Jabi-Tehnan districts of Amhara regional state, Western Ethiopia. It is bordered on the South East by Dembecha district, on the west by Bure district, on the northwest by Sekella district, on the north by Quarit district, and on the east by Dega Damot. The capital town is Finote-selam, which is about $387 \mathrm{~km}$ from the National capital, Addis Ababa and 176km South West of Bahir Dar town. The population of the district was 211,516 in 2017 with an average annual growth rate of $2.8 \%$ [32]. The district is divided into 38 kebeles, which is the lowest administrative units of the country, and three town administrations. More than $90 \%$ of the people in the district live in rural areas practicing mixed farming.

The altitude of the district ranges from 900 to 2,300 meter above sea level. Much of the area lies in the higher altitude range, closer to 2,300 meters. Agro-ecologically, $88 \%$ of the district is classified as mid land and the remaining $12 \%$ as low land. The topography of the district is dominated by areas of flat plain. According to Asmare \& Gure, 2019 [33], the topography is classified as 65\% flat plain, 15\% mountainous, $15 \%$ undulating and $5 \%$ valley. The rainfall distribution is uni-modal and the rainy season 
lasts for four months from mid-May to mid-September. The annual rainfall ranges 1,250 $\mathrm{mm}$ per annum. The mean minimum and maximum temperatures are $140 \mathrm{c}$ and $320 \mathrm{c}$, respectively [33]. According to the report compiled from 17 kebeles of the district, malaria disease continued to be major health threat resulting in total morbidity of 4,345, and average annual parasite rate (API) of 11.23 (Tsehaye 2018, personal communication). A cross sectional active malaria prevalence survey from randomly selected kebeles of the district in 2013 showed the disease prevalence of 2.8\% [34].

A total of 500 metal-roofed houses constructed with mud walls, and without screening, with at least one child aged 6 months to 14 years, will be selected. Consent will be sought from the parents or guardians for them to join the study by field assistants (Additional file 1).

\subsection{Study participants}

\subsubsection{Population}

The source population of this study will be the total population found in Jabi Tehnan district, Amhara regional state, Northwest Ethiopia

\subsubsection{Eligibility criteria}

The study population will be all households that are found in the rural areas of Jabi-Tehnan district having at least one child (under 14 age) in their family.

\subsubsection{Sample size rationale}

\section{Clinical}

Studies conducted in Ethiopia[12] and Gambia [9, 10, 26, 35] suggested that houses with their doors and windows screened have resulted in reduction of mosquito density measured between 40 to $70 \%$ as compared to control houses by implication leading to malaria case reduction. Thus, considering the experiences from both countries this design is developed to measure $50 \%$ reduction of malaria in the study villages. In addition, a study conducted by Ayalew et al., (2016) [34] in 3 villages in the district showed the $2.8 \%$ prevalence which is used as a benchmark for this trial. Thus, the sample size was determined following the guideline for cluster randomized trials developed by Hayes \& Bennett (1999) [36], which accounts the intra-cluster correlation coefficient (ICC), incidence rate, the expected effect and the power of the study. Accordingly, 500 households, will be selected from 30 villages, to detect a $50 \%$ reduction in malaria cases with more than $80 \%$ power at the $5 \%$ significance level.

\section{Entomological}

Preliminary entomological collections made between July to Dec 2019 showed an average $2.8(S D=6.87)$ An. gambiae s.I. mosquitoes/per trap night. Therefore, we will use this data as a baseline and propose to reduce the mean number of mosquitoes captured per trap night by $50 \%$. Fifty houses from each 
treatment will be used to demonstrate a $50 \%$ reduction in indoor-entering mosquitoes associated with housing screening, with $80 \%$ power at the $5 \%$ level of significance.

\section{Social}

We will use the baseline data from the 3,010 households collected in the district for the socioeconomic analysis. The sample size is randomly selected from a fresh list of households in the rural and semiurban part of the district. We will follow these households and collect additional information two times (midline and end line surveys).

\subsubsection{Recruitment}

For this study, the principal investigator (AA) will base in the study district and form a recruitment committee. The committee will be composed of the head of local administration, representative from village HEWs, the head of local health center and the principal investigator (AA). The team will make house to house visit and explain the objective of the study to the candidate household heads. The list of candidate households will be separately generated from the roster containing the list of household heads of the district by the data manager (ZA). In instances where the candidate household refuses to participate, reserve candidate households will be invited to participate.

\subsection{Design}

\subsubsection{Type of Study Design}

A longitudinal study of four-armed household clustered randomized control trial will be conducted to estimate the incremental benefit of combining house screening, long-lasting insecticidal nets and pushpull technology.

\subsubsection{Study flow chart}

The study design is summarized in a flow chart as shown in Fig. 1. The study contains four treatments randomly assigned to households with village as the blocking factor. As some houses get screening and others not, there could be a risk of diverting potentially infectious mosquitoes from screened houses to unscreened ones. Thus, to avoid such risks care will be taken to not exceed $5 \%$ of houses per village as it was recommended from previous studies from Gambia[14]. Studies conducted in other countries confirmed that the risk of mosquito spread to unprotected houses is unlikely to increase if the proportion of houses protected is less than $10 \%[5,14]$.

In this study, a total of $167,246,250$ and 175 houses will be included in four different treatments, treatment 1 (control), treatment 2 (LLINs and HS), treatment 3 (LLINs, HS and PPT) and treatment 4 (LLINs and PPT), respectively. One resident child will be enrolled from each house and followed up for two consecutive years. If a child moves or quits the study, we replace the child from the same house. In 
instances where the whole family leaves the area, we will document them as drop outs from the follow up.

The selected children will be checked up every month for clinical symptoms of malaria infection, splenomegaly, and development of anaemia. Blood test will be conducted if the child develops clinical symptoms. The child will also be surveyed th the beginning and end of each transmission season to estimate the prevalence of Plasmodium species infection, parasite density, and the prevalence of anaemia. Fifty houses (50) from each treatment arm will be randomly selected for seasonal entomological monitoring.

\subsubsection{Sequence generation}

As described in the Section 3.3.2, all the households in the district are eligible to the treatment. For the purpose of this study, we divided the 30 kebeles into 66 sub-kebeles. In each sub-kebele, we obtain a fresh

list of farmers, which are organized into one-to-five groups. ${ }^{[1]}$ The houses in each sub-kebele are randomly assigned into the four treatment arms.

[1] The one-to-five groups are the lowest units of farmers' organizations that five farmers and a chair person organize themselves into a group. As of April 2019, The Jabi-tehnan district had a total of 5,608 one-to-five-groups of farmers.

\subsubsection{Randomization}

As the study kebeles are scattered across different localities (and elevations) of the district, the risk of malaria infection also varies among kebeles (document review from district malaria information). Therefore, houses will be first clustered into kebeles and then into sub-villages before randomly assigned into the study arms. The randomization process will be conducted using the stata's sampsi package and efforts will be made to ensure that each kebele receives balanced number of treatments following the standard protocol developed by Pinder et al (2016) [14]. Stratified randomization by sub-kebeles will take out the kebele effect and the likelihood of chance imbalances between study arms. All entomological, epidemiological, and social studies will be conducted following the same rule described above i.e, stratified randomization. The randomization will be done by ZA to prevent selection bias by concealing the allocation sequence from the field researchers assigning kebeles into the four treatment arms until the moment of assignment. Thus, both the chief investigator and the principal investigator will not be involved in the randomization process.

\subsubsection{Blinding}

Screening of windows and doors will not be blinded as it is difficult to conceal them. However, we will follow the world health organization 2015 [37] guideline in blinding other activities. The blood films will be read by microscopists blinded to the identity and intervention status of the subjects. Mosquito collection will be made by using standard light traps thereby avoiding the potential bias that could be 
introduced by the fieldworkers to collect specimens. Mosquito identification will be made by different technicians who will not know the trap location.

\subsection{Interventions}

\subsubsection{Long-lasting Insecticidal nets}

DuraNet ${ }^{\circledR}$ (Shobikaa Impex pvt Ltd, Karur, Tamil Nadu 639006, India) will be provided to all households (treatment and control) at the rate of one bed net for two people following the NMCP and per WHO recommended universal net coverage $[1,38]$. The nets will be provided by NMCP district office and will be distributed to study households in the first week of July 2020. All study participants including the control group will receive new LLINs free of charge at the beginning of the intervention regardless of the previous ownership, with householders maintaining their existing nets at the time of distribution.

\subsubsection{House screening}

The house screening work will be undertaken by pre-trained artisans of about 4 to 5 people to be recruited from the study area. The training will be done by an in-country contractor familiar with screening of houses. The screening of 500 houses is expected to take about 2 months. Household owners will be trained on the care needed to keep the screens intact and effective and avoid activities that could result into making holes in the mesh or cause the screen to slide and create spaces that could allow mosquito entries into the houses. Other routine procedures to be used to reduce mosquito entry into houses such as closing windows and doors early will be emphasized and adherence to this practice monitored passively by the study team. As part of the intervention all the windows, doors and eaves will be screened using polyethylene material (POLYTEX INTERNATIONAL (UK) LIMITED, 14 Rutherford Way, Drayton Fields, Daventry, Northants, NN11 8XW, United Kingdom).

\subsubsection{Push-pull technology}

Push pull technology is a biological method of controlling pests of cereals: stem borer and striga. It is a novel technology in which a repellent intercrop and attractant trap plant is used simultaneously. The stem borers are repelled from the food crop and simultaneously attracted to a trap crop. It has three components which include the push, the pull, and the intercropped plant. The push component refers to desmodium plant. The pull component refers to the brachiaria grass which is usually planted at the periphery of the plot. While the desmodium is rich in protein, the brachiaria is rich in carbohydrate, which will serve as an important source of animal feed. The detail application of PPT is described elsewhere [39]. Thus, as part of the intervention training will be provided to selected farmers on the concept of PPT and how to use it. This include plot preparation, sowing, establishing, managing, animal feed preparation and seed harvesting. A total of 250 farmers who successfully completed the training and whom been selected to house screening treatment will be provided PPT plant seeds namely Mulato II Hybrid Brachiaria, (3kg/ha, Barenbrug, 26 Prosperity Way Dandenong South VIC 3175, Australia) and 
Desmodium intortum 3-5kg/Ha from the same company. The amount of $\mathrm{Kg}$ provided to each farmer will be decided on the size of plot made ready by the farmer.

\subsubsection{Risks and harms}

There is no risk of any infection as we do this study, however, finger pricking for blood test is usually with mild pain and discomfort.

\subsection{Data Collection and Management}

\subsubsection{Study endpoints/outcomes}

\section{Clinical evaluations}

The main outcomes of the clinical study will be the malaria incidence, splenomegaly, and anemia. Thus, the baseline clinical survey of all study children will take place between Sept/Oct 2020 to determine the clinical parameters. As part of the follow up, starting from Nov 2020, trained health extension workers will visit the house of each child and take data of clinical symptoms, temperature, and splenomegaly (sign of spleen enlargement). While monthly follow up of the child continues throughout the two years of study period (Sept 2020 to Dec 2022) for documentation of any malaria incidence, blood testing of the entire cohort of the study children will be repeated at the end of each rainy season (Sept/Oct 2021 and) and Sept/Oct 2022. In between the annual clinical surveys (i.e. at the time of monthly follow up), blood testing will be done only to children who develop fever to minimize community fatigue development.

Temperature measurement, RDT testing, blood haemoglobin measurement, blood sample collection for microscopy will be conducted by a licensed laboratory professional following the WHO guideline [37].

\section{Entomological evaluations (medical and Agricultural pests)}

The major entomological outcomes of the study are change in mosquito density, rate of mosquito infection (Sporozoite rates), and the entomological inoculation rate (EIR). Both Indoor and outdoor mosquito collections will be made using the Centers for Disease Control (CDC)'s light traps to estimate the mosquito density. Collections will be made twice per year (July to Sept and January to March) starting from July 2020 to Oct 2022. All mosquito collections will be preserved in silica gel and transported to the International Centre of Insect Physiology and Ecology (ICIPE) headquarters where they will be identified to species level and examined for sporozoite infection using Polymerase chain reaction (PCR) technique. Blood meal analysis of fed females will be done using Enzyme linked immunosorbent assays (ELISA). In line with the investigation of vectors of medical entomology, the agronomics team will evaluate the efficacy of PPT against stem borers, fall armyworm (FAW) and striga infestation. Visual observation will be made in selected plots from each treatment arm (PPT plot and control) and the degree of infestation will be measured using scale scores where 1 attributes to clean plant with no infestation and 5 attributes to very severely damaged plant [40].

\section{Economic and social science evaluations}


The key primary outcomes of the social sciences component of the impact evaluation, including the cost of malaria burden and cost effectiveness of house screening, are registered in a separate social science registry [41].

\subsubsection{Data collection methods}

For this study, each household will be provided with a specific identification (ID) number. The child who included in the study from each household will receive a unique personal, three-digit ID number (village number/household number/person number). All forms and datasets will identify participants by their unique identifier numbers, and names will not be used. The geographic coordinates of all study households will be documented at the beginning of the study and used in mapping the study points. Questionnaires and forms used will be initially prepared in English and translated into Amharic for data collection and then it will be translated back to English later. Databases will be password protected and accessible only to authorized personnel. All documents will be securely stored in locked filing cabinets and accessible only to authorized personnel.

\section{Epidemiological data collections}

The main morbidity outcome will be the incidence of clinical malaria assessed by active infection detection documented during the fortnight house to house visit over the course of the two-year study period. The axillary temperature will be taken fortnightly by CHWs from all enrolled study children and if the child shows $\geq 37.5^{\circ} \mathrm{C}$ or history of fever in the past 48 hours, then, a rapid diagnostic test (RDT, CareStart ${ }^{\circledR}$ Malaria Pf/Pv combo test; Access Bio, Inc., Somerset, NJ, USA) will be conducted to further cross check the presence of malaria parasite. In line with this, blood spot sample (DBS) will be collected for later testing using PCR-based methods. Malaria positive children identified by RDT during the crosssectional surveys will be provided with a full dose of Artemether-lumefantrine (AL) and chloroquine by a trained health worker following national guidelines. In Ethiopia, $\mathrm{CHWs}$ are trained and mandated to test and treat malaria. The second epidemiological outcome will be the prevalence of anaemia among the study participants (children) and thus, all children between 6-59 months of age will be graded according to their $\mathrm{Hb}$ level.

\section{Entomological data collection}

A total of 8 houses, (four houses for indoor and four houses for outdoor LTs) catches will be selected from each study village. Indoor and outdoor catches will be collected from each study arm. Mosquitoes will be collected indoor and outdoor from 6:00 pm to 06:00 am from each selected house using standard battery-operated CDC light traps. Traps will be hung from the ceiling or from roof support at the foot end of the bed where people sleep at night and each trap will be suspended about 1.5 meters from the floor. Traps will be also hung outdoor under the eaves of the house for outdoor mosquito collection. Each trap will be set by trained research team members. Collection bags will be retrieved from traps in each house in the morning between 06:00 am and 07:00 am. All unfed, fed, half gravid and gravid adult female Anopheles mosquitoes (Indoor and Outdoor) will be identified using taxonomic keys of Gilles and Coetzee 
(1987) [42] . Dried head and thorax of Anopheles gambiae s.I., and Anopheles pharoensis collected by LTs from each study villages will be carefully separated from the abdomen and tested for $P$. falciparum and, P. vivax-210 and P. vivax-210 circumsporozoite proteins (CSPs) simultaneously following the protocol developed by Beier et al. 1988 [43].

\section{Socio-economic data collections}

Semi-structured questionnaire will be prepared to collect socio-demographic data at the beginning and end of the study. Each study household will be observed for durability of the screening material, the presence of holes, tears, losses and the fitness of windows and doors. The presence or absence of LLINs and the number and age of people who slept under bed nets in the previous night will be assessed in every visit to mosquito sampling (before and after screening). The impact of screening intervention on bed net use rate will be measured by observing bed net use among household members before and after house screening intervention, and between intervention and control groups. For the social science component of this impact evaluation, the team will collect data using household surveys, focus group discussions and key informant interviews. A baseline survey, midline survey and end line surveys will be undertaken using a structured household survey questionnaire and trained enumerators.

\subsubsection{Data management}

Data will be stored in two forms, i.e., hard and soft copies in compliance with the principles of good clinical practice protecting the confidentiality of participants. Specimens of mosquitoes, blood film slides and genotype print of PCR outcomes will be maintained by the principal investigator and be available up on request from authorized representatives, regulatory bodies. The results of the study will be made publicly available through peer reviewed journals.

\subsection{Statistical methods}

\subsubsection{Statistical methods}

\section{Malaria Incidence Data analysis}

Change in clinical malaria incidence over two years period among the treatment and control arms will be determined using Poisson distribution. Mixed effects Poisson model will be used to test the difference in incidence rate among the study arms, to determine effects of the repeated measurements within house, village, the effect of year and village-intervention interaction effects. To control the effect of clustering or village and individual level confounding factors such as gender and age, these covariates will be fitted in to random effects during analysis. If a child is diagnosed with malaria case within 28 days of the first episode with the same plasmodium species, then it will be put on treatment as part of the safety protocol, however, the case will not be included in the analysis. The prevalence of anaemia among the study participants (children) will be analysed using the guideline developed by world health organization [44]. Accordingly, all children between 6-59 months of age, whose $\mathrm{Hb}$ level is recorded between 10.0-10.9 $\mathrm{g} / \mathrm{dl}$, 8.0-9.9 g/dl and less than $8.0 \mathrm{~g} / \mathrm{dl}$ will be graded as mild, moderate, and severe, respectively. In line with 
this we will also report community level anaemia prevalence. Thus, a prevalence of anaemia will be stated as severe if it is documented over $40 \%$ (combining mild, moderate, and severe) and moderate if the prevalence is $20-39.9 \%$. Both malaria and anaemia prevalence data will be compared in the intervention and control houses using multilevel mixed-effects logistic regression models, taking village effects into account.

\section{Entomological data analysis}

Indoor and outdoor Anopheles densities will be compared for each study arm using a student t-test. Overall mosquito density among treatment arms will be compared using one-way analysis of variance (ANOVA) and if there is significance difference among treatment arms mean separation test will be done using turkey's range test. The sporozoite rate will be determined as the proportion of malaria vectors positive for CSPs over the total number tested for CSPs.

The durability test of screening material will be conducted following the guideline developed by Kinde et al. (2018) [45] and modification of world health organization [46] guideline for evaluation of durability of long-lasting insecticidal mosquito nets under operational conditions. Accordingly, the durability of the screening intervention will be measured by assessing the number of holes on the meshes. Fabric integrity of all HSs fixed into windows and doors will be assessed for holes at each monitoring round. The proportion of HSs with any holes will be presented with total number of HSs in surveyed households as denominator. The scale of HS damage will be quantified using hole index formula recommended for mosquito nets and it is given as Hole index $=(A \times$ no. of size -1 holes $)+(B \times$ no. of size- 2 holes $)+(C \times$ no. of size-3 holes $)+(D \times$ no. size-4 holes). Numbers 1, 2, 3, 4 refer to the size of the whole, letters A, B, C, D refer to the weight of the hole and given as 1,23, 196 and 578 [46]. The fabric integrity of screens fixed in doors will be analysed separately as its exposure to wear and tear is higher as compared to that of windows. In depth interviews, photo-based observation will be conducted in order to measure community acceptance of the intervention.

\section{Social science data analysis}

The socioeconomic data analysis will be done using descriptive and econometric approaches. An incremental cost effectiveness ratio (ICER), will be calculated for each outcome and arm using standard Disability Adjusted Life years (DALYs). The interventions will be ranked according to cost effectiveness, whereas inequality in terms of health outcomes, will be measured by the Gini coefficient and the concentration index.

\subsubsection{Handling of dropouts/withdrawals}

The right of participants to withdraw from the study at any time without giving a reason will be communicated prior to recruiting each participant. If the first child from the house is moved to other place for various reasons, the second child will be replaced. In unlikely scenario of the situation in which the entire family moves (e.g. building home in nearby town is a common practice in the area), the follow up 
will be discontinued and other related data collection activities (entomology and social science) will be replaced by reserve house.

\subsection{Safety and monitoring}

\subsubsection{Safety evaluations}

House to house visit, children follow up and RDT testing during monthly follow up period will be conducted by the trained health extension workers (HEWs). Yearly blood sample collection and processing will be handled by profession laboratory technicians. Blood samples for RDTs, microscopic examination and Haemoglobin test measurements will be collected using aseptically disposable lancets. Positive, cases found during the monthly follow up will be treated by the HEWs according to the national guidelines [47] and any complications that could potentially develop in to series adverse effect (SAE) on participants will be referred to the nearby health center or hospital and reported to the principal investigator.

\subsubsection{Trial Oversight}

The implementation of this clinical trial will be overseen by Amhara regional public health institute (partner and legal body which ensures the proper implementation of ethical protocols) and NORAD, sponsoring the trial. A contract agreement will be signed among the three parties (the PIs, the sponsors, and the regulatory partners). As part of the contract the Pls will submit annual reports detailing the progress of the trial, the safety procedures put in place and the overall impact of the study. Blood sample collection and treatments are part of routine malaria control in Ethiopia and will be undertaken in collaboration with the health workers at the health posts and therefore, no need to oversee the routines. Side wastes that result from the trial such as used insecticide nets, empty sachets, cartons, plastic bags, used gloves, pricking needles and other contaminated materials will be properly disposed following the guideline set by Robertson et al. (1995) [47].

\subsubsection{Ethics approval}

The study was approved by the IRB of the Amhara public health Institute, Amhara reginal state (ref: APHI/HRTTD/03/341/2019). The protocol was registered online on Date 28/05/2020 on sight www.pactr.org With registration No: PACTR202006878245287.

\section{Timelines of activities}




$\begin{array}{llllllllllllll}\text { Activity by year and month } & \text { J } & \text { F } & M & \text { A } & M & J & J & A & S & 0 & \text { N } & \text { D }\end{array}$

2019

Project submission to IRB and approval $\quad X \quad X \quad X \quad X$

Development of data collection tools

$\mathrm{X}$

Sensitization of study population

Census and mapping

$x \quad x$

Epidemiological and entomological pilot

study

\section{0}

Selection of study villages

Randomization

Procurement of Screening material

Procurement of Desmodium \& Brachiaria seed

Seed distribution and plantation

Screening of selected houses

Protocol registration

Epidemiological \& entomological Data collection

Social studies data collection

Cost effectiveness data collection

2021

Malaria prevalence survey

entomological Data collection

Entomology lab assays

Annual social studies survey

Data entry and cleaning

Cost effectiveness study

\section{2}

Malaria prevalence survey

entomological Data collection

$$
\mathrm{X} x
$$

$x$

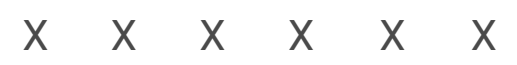




\begin{tabular}{|c|c|c|c|c|c|c|c|c|c|c|c|c|}
\hline Entomology lab assays & & & & & & & & & & & $x$ & $x$ \\
\hline Annual social studies survey & & & & & $x$ & $x$ & $x$ & $x$ & $x$ & $x$ & $x$ & \\
\hline Data entry and cleaning & $x$ & $x$ & $x$ & $x$ & $x$ & $x$ & $x$ & $x$ & $x$ & $x$ & $x$ & \\
\hline Cost effectiveness study & & & & & $x$ & $x$ & $x$ & $x$ & $x$ & $x$ & $x$ & $x$ \\
\hline $\begin{array}{l}\text { Dissemination of findings to the study } \\
\text { community }\end{array}$ & & & & & & & & & $x$ & $x$ & $x$ & $x$ \\
\hline $\begin{array}{l}\text { Publication and final report to stake } \\
\text { holders }\end{array}$ & & & & & & & & & $x$ & X & $x$ & $x$ \\
\hline
\end{tabular}

\section{Discussion}

Countries and inter-state actors who work in public health sectors are running out of options to contain vectors of malaria vectors as existing interventions become less effective due to growing insecticide resistance [4] and adaptive behavioral shift of vector mosquitoes to evade the interventions [48]. Thus, all options including research for novel vector control interventions [49], combination of existing strategies (e.g. combination of LLINs and IRS, [50] and supplementing them with auxiliary strategies e.g. combination of LLINs and HS, [14] are being tried as part of the ongoing research and innovation in the field of vector control. In addition, a recent modelling study conducted by Kassie et al. (2020) [51] showed that integrated interventions aimed at relaxing the constraints of multiple health problems (animalhuman-plant health) are more effective in improving the overall welfare of humans as compared to the stand alone interventions. Thus, in this study we aim to test and assess the combined effect of 1) LLINs and HS 2) LLINs, HS and PPT and 3) LLINs and PPT. Households that receive LLINs alone will be used as control.

The first outcome of this trial will be the indicators of clinical malaria (malaria incidence, splenomegaly, and anaemia) documented among the 500 children enrolled in this study. Thus, 500 children under 14 years of age will be enrolled to the study cohort and followed up for two years. Follow up will be made every month by health extension workers who also conduct routine health care activities at village level. A study conducted in selected villages of the district by Ayalew et al., [34] shows that malaria prevalence in the area is relatively low with prevalence rate of $2.8 \%$. We will use this study as a benchmark to assess the impact of our intervention. As part of the study, all households participate in this study will get new bed nets freely based on WHO recommendation of universal coverage. The second outcome of the study will be entomological indicators (vector species composition, vector mosquito density, infectivity rate, feeding rate). Thus, mosquito collection will be done every month from selected 150 houses distributed over 30 villages using $C D C$ and samples will be processed following standard protocol [52]. The third outcome of the study will be the durability test of screening material and an incremental cost effectiveness ratio which will be conducted following the guideline developed by Kinde et al., [45] and World Health Organization [46] guideline for evaluation of durability of long-lasting insecticidal mosquito nets under operational conditions. 
Screening houses for the purpose of excluding malaria vector mosquitoes in to residential structures is well established practice in Europe [53], America [54] and some parts of tropics [5] however, this experiences were given less emphasis in Africa as main stay vector control intervention (LLINs and IRS) predominated the vector control arena. A specific design of the house (e.g. thatched roof, Iron sheet cover, closed eve, open eve) can play critical role in increasing or decreasing the odds of malaria infection [9] and mosquito density per capita or per house $[35,55,56]$. In addition to alleviating malaria, the introduction of house screening intervention in houses has resulted in reduced infection of other vector borne diseases such as dengue, leishmaniasis, yellow fever and Zika [57].

Push pull technology on the other hand is a semio-chemical based intervention in which two plant species are being used in integrated approach with primary objective of increasing the quality and quantity of crop yield per unit area. It is developed in icipe research center in collaboration with Rothamsted research center UK and scaled up throughout Africa to support smallholder farmers. The strategy is non-synthetic chemical, and it involves the intercropping of cereal crops with a forage legume, desmodium, and planting Napier grass as a border crop [23]. In this approach the Desmodium intortum plant releases the semio-chemical which repels stem borer moths, and attracts their natural enemies, while Napier grass (now replaced by Bracharia mulatotu-II) attracts the pest. In addition, the Desmodium plant can effectively suppress striga weeds and improve soil fertility through nitrogen fixation. Both plants provide animal fodder thereby increasing the health and productivity of livestock.

Commercialization of these companion crops can be used as a source of income generation through seed production. By integrating the push-pull technology and malaria vector control interventions, we hypothesize that the PPT will improve the livelihood of farmers through increased production and productivity thereby the synergy of the technologies contributing to improved overall life standard including the fight against malaria.

We expect some mild risks arising from this study. Screening of windows and doors may lead to reduced aeration in houses which may result in respiratory infection of children as it was hypothesized in similar studies conducted in the Gambia [14]. Routine monitoring of respiratory infections will be made on households enrolled in the study to document if there exist any incidence. In case of encountering any such infections, children will be referred to nearby health center for immediate treatment. We hypothesize that there would be significant reduction in mosquitoes entering in to houses due to screening which may lead to complacence in using bed nets. As part of routine monitoring and house to house visit, we will make sure that people should continue to use LLINs as usual and educate them that HS is not standalone intervention.

People involved in this study will be benefited from freely available house screening materials and push pull technology. Their houses (windows and doors) will be screened and they will be trained how to use the push pull technology. As part of the technology transfer each farmer will be provided with training and seeds of Desmodium and Bracharia. Agricultural development agents (DAs) will visit each household regularly to support the establishment of the technology on their farm. In addition, children enrolled in this study will be benefited from regular check-up and treatment whenever there is malaria incidence. 
This trial will provide critical information on whether combining LLINs, HS and PPT will have additive value in malaria control efforts. This study will also generate useful information on cost effectiveness of the intervention in combination and when applied singly. In addition, the acceptability of both HS and PPT interventions by the community will be carefully assessed. Thus, the findings of this study will be used for an effective planning and implementation of vector control interventions by national malaria control programs.

\section{Declarations}

\section{Protocol version}

Dummy: 17 July 2020, version 2.0.

\section{Status}

The trial is currently recruiting.

\section{Additional files}

Additional file 1: Ethics information sheet and consent form.

Additional file 2: Schedule of enrolment, interventions, and assessment.

Additional files 1 and 2 are not provided in this version

\section{Abbreviations}

$\mathrm{CDC}$, Centers for Disease Control and Prevention; CV, coefficient of variation; EIR, entomological inoculation rate; $\mathrm{Hb}$, hemoglobin; LLIN, long-lasting insecticidal nets; PCR, polymerase chain reaction; PI, principal investigator; RDT, rapid diagnostic test; WHO, World Health Organization

\section{Acknowledgements}

This study is supported by the Norwegian development Cooperation, NORAD. We would like to extend our heartfelt thanks to Amhara regional malaria control program for freely availing the LLINs needed for this study.

\section{Authors' contributions}

$A A, M K, Z A$, and $C M$, conceived and designed the study, and drafted the manuscript. AA designed the analytical plan. BE and AB helped in designing the PPT component. POS, helped in designing the house screening component. DT involved in designing the molecular study part of the method. All authors read and approved the final manuscript.

\section{Authors' information}


CM is the chief investigator and AA is the principal investigator in Ethiopia. AA, CM and POS are medical entomologists. MK and ZA are economists. BE is an agronomist. DT is molecular biologist. The study sponsor and funders do not play a role in study design; collection, management, analysis, and interpretation of data; writing of the report; and the decision to submit the report for publication.

\section{Competing interests}

All authors declare that they have no competing interest.

Trial Registration no: PACTR202006878245287.

Funding: Norwegian Agency for Development Cooperation (NORAD) through the project Combating Arthropod Pests for Better Health, Food and Resilience to Climate Change (CAP-Africa) (Project Ref No: )

\section{Trial Registration no}

PACTR202006878245287.

\section{Funding}

Norwegian Agency for Development Cooperation (NORAD) through the project Combating Arthropod Pests for Better Health, Food and Resilience to Climate Change (CAP-Africa) (Project Ref No: )

\section{References}

1. WHO. World malaria report 2019. 2019. www.who.int/malaria. Accessed 7 Aug 2020.

2. WHO. Global technical strategy for malaria 2016-2030. 2015. http://apps.who.int/iris/bitstream/10665/176712/1/9789241564991_eng.pdf?ua=1.

3. Antoine Sanou; Luca Nelli; W.Moussa Guelbeogo; Fatoumata Cisse; Michel M. Tapsoba; Pierre Ouédraogo; N'falé Sagnon; Hilary Ranson JMHM, Ferguson. Insecticide resistance outpaces behavioural adaptation, as a response to Long-Lasting Insecticidal Net distribution, in malaria vectors in Burkina Faso. Res Sq. 2021.

4. Ranson $\mathrm{H}$, Lissenden N. Insecticide Resistance in African Anopheles Mosquitoes: A Worsening Situation that Needs Urgent Action to Maintain Malaria Control. Trends Parasitol. 2016;32:187-96.

5. Lindsay SW, Emerson PM, Charlwood JD. Reducing malaria by mosquito-proofing houses. 2002;18:510-4.

6. Lindsay SW, Jawara M, Paine K, Pinder M, Walraven GEL, Emerson PM. Changes in house design reduce exposure to malaria mosquitoes. 2003;8:512-7.

7. Kirby MJ, Njie M, Dilger E, Lindsay SW. Importance of eaves to house entry by anopheline, but not culicine, mosquitoes. J Med Entomol. 2009;46:505-10.

8. Gimnig JE, Slutsker L. House screening for malaria control. Lancet. 2009;374:954-5. doi:10.1016/S0140-6736(09)61078-3. 
9. Jatta E, Jawara M, Bradley J, Jeffries D, Kandeh B, Knudsen JB, et al. How house design affects malaria mosquito density, temperature, and relative humidity: an experimental study in rural Gambia. Lancet Planet Heal. 2018;2:e498-508. doi:10.1016/S2542-5196(18)30234-1.

10. Jawara M, Jatta E, Bell D, Burkot TR, Bradley J, Hunt V, et al. New Prototype Screened Doors and Windows for Excluding Mosquitoes from Houses: A Pilot Study in Rural Gambia. Am Soc Trop Med Hyg. 2018;99:1475-84.

11. Ogoma SB, Lweitoijera DW, Ngonyani H, Furer B, Tanya L, Mukabana WR, et al. Screening Mosquito House Entry Points as a Potential Method for Integrated Control of Endophagic Filariasis, Arbovirus and Malaria Vectors. 2010;4:1-8.

12. Massebo F, Lindtjørn B. The effect of screening doors and windows on indoor density of Anopheles arabiensis in south-west Ethiopia: a randomized trial. 2013;:1-6.

13. Getawen SK, Ashine T, Massebo F, Woldeyes D, Lindtjørn B. Exploring the impact of house screening intervention on entomological indices and incidence of malaria in Arba Minch town, southwest Ethiopia: a randomized control trial. Acta Trop. 2018;181:84-94.

14. Pinder M, Conteh L, Jeffries D, Jones C, Knudsen J, Kandeh B, et al. The RooPfs study to assess whether improved housing provides additional protection against clinical malaria over current best practice in The Gambia: Study protocol for a randomized controlled study and ancillary studies. Trials. 2016;17:1-11. doi:10.1186/s13063-016-1400-7.

15. Pinder M, Bradley J, Jawara M, Affara M, Conteh L, Correa S, et al. Articles Improved housing versus usual practice for additional protection against clinical malaria in The Gambia ( Roo Pf s ): a household-randomised controlled trial. Lancet Planet Heal. 2021;5:e220-9. doi:10.1016/S25425196(21)00002-4.

16. West PA, Protopopoff N, Wright A, Kivaju Z, Tigererwa R, Mosha FW, et al. Indoor Residual Spraying in Combination with Insecticide-Treated Nets Compared to Insecticide-Treated Nets Alone for Protection against Malaria: A Cluster Randomised Trial in Tanzania. PLoS Med. 2014;11.

17. Protopopoff N, Wright A, West PA, Tigererwa R, Mosha FW, Kisinza W, et al. Combination of insecticide treated nets and indoor residual spraying in northern Tanzania provides additional reduction in vector population density and malaria transmission rates compared to insecticide treated nets alone: A randomised control trial. PLoS One. 2015;10:1-11.

18. Loha E, Deressa W, Gari T, Balkew M, Kenea O, Solomon T, et al. Long-lasting insecticidal nets and indoor residual spraying may not be sufficient to eliminate malaria in a low malaria incidence area: Results from a cluster randomized controlled trial in Ethiopia. Malar J. 2019;18:1-15. doi:10.1186/s12936-019-2775-1.

19. Corbel V, Akogbeto M, Damien GB, Djenontin A, Chandre F, Rogier C, et al. Combination of malaria vector control interventions in pyrethroid resistance area in Benin: A cluster randomised controlled trial. Lancet Infect Dis. 2012;12:617-26.

20. Hawkes C, Ruel M. Policy and Practice The links between agriculture and health: an intersectoral opportunity to improve the health and livelihoods of the poor. 2006;025650. 
21. Badiane 0 , Ulimwengu J. Malaria incidence and agricultural efficiency in Uganda. Agric Econ (United Kingdom). 2013;44:15-23.

22. Kassie M, Stage J, Diiro G, Muriithi B, Muricho G, Ledermann ST, et al. Push-pull farming system in Kenya: Implications for economic and social welfare. Land use policy. 2018;77:186-98.

23. Khan ZR, Midega CAO, Pittchar JO, Murage AW, Birkett MA, Bruce TJA, et al. Achieving food security for one million sub-Saharan African poor through push-pull innovation by 2020 . Philos Trans $R$ Soc $B$ Biol Sci. 2014;369.

24. Pickett JA, Woodcock CM, Midega CAO, Khan ZR. Push-pull farming systems. Curr Opin Biotechnol. 2014;26:125-32.

25. Kebede Y, Baudron F, Bianchi F, Tittonell P. Unpacking the push-pull system: Assessing the contribution of companion crops along a gradient of landscape complexity. Agric Ecosyst Environ. 2018;268 July:115-23.

26. Kirby MJ, Ameh D, Bottomley C, Green C, Jawara M, Milligan PJ, et al. Effect of two different house screening interventions on exposure to malaria vectors and on anaemia in children in The Gambia: a randomised controlled trial. Lancet. 2009;374:998-1009. doi:10.1016/S0140-6736(09)60871-0.

27. Chepchirchir RT, Macharia I, Murage AW, Midega CAO, Khan ZR. Ex-post economic analysis of pushpull technology in Eastern Uganda. Crop Prot. 2018;112 June:356-62.

28. icipe. Push-Pull-a novel farming system for ending hunger and poverty in sub-Saharan Africa. International Centre of Insect Physiology and Ecology (icipe). 2019.

29. Sternberg ED, Cook J, Ahoua Alou LP, Aoura CJ, Assi SB, Doudou DT, et al. Evaluating the impact of screening plus eave tubes on malaria transmission compared to current best practice in central Côte d'Ivoire: A two armed cluster randomized controlled trial. BMC Public Health. 2018;18:1-13.

30. Menger DJ, Omusula P, Wouters K, Oketch C, Carreira AS, Durka M, et al. Eave Screening and PushPull Tactics to Reduce House Entry by Vectors of Malaria. 2016;94:868-78.

31. Tsion T. Kidane \& Steven H. Worth. Different Agricultural Extension Systems Implemented in Africa: A Review. J Hum Ecol. 2016;55:185-193.

32. CSA. Population Projection of Ethiopia for All Regions At Wereda Level from 2014 - 2017. Addis Ababa; 2013. doi:10.1186/1746-4269-3-28.

33. Asmare MT, Gure A. Effect of exclosure on woody species diversity and population structure in comparison with adjacent open grazing land: the case of Jabi Tehnan district north western Ethiopia. Ecosyst Heal Sustain. 2019;5:98-109. doi:10.1080/20964129.2019.1593794.

34. Ayalew S, Mamo H, Animut A, Erko B. Assessment of current malaria status in light of the ongoing control interventions, socio-demographic and environmental variables in Jiga area, northwest Ethiopia. PLoS One. 2016;11:1-10.

35. Lindsay SW, Jawara M, Mwesigwa J, Achan J, Bayoh N, Bradley J, et al. Reduced mosquito survival in metal-roof houses may contribute to a decline in malaria transmission in sub-Saharan Africa. Sci Rep. 2019;9:1-10. 
36. Hayes RJ, Bennett S. Simple sample size calculation for cluster-randomized trials. 1999;:319-26.

37. WHO. Guidelines for treatment of malaria. 3rd edition. Geneva, Switzerland: World Health Organization; 2015.

38. PMI. Ethiopia Malaria Operational Plan FY 2019- President's Mlaria. Addis Ababa: PMI-USAID; 2019.

39. Khan ZR, Pickett JA, Wadhams LJ, Hassanali A, Midega CAO. Combined control of Striga hermonthica and stemborers by maize-Desmodium spp. intercrops. Crop Prot. 2006;25:989-95.

40. Hailu G, Niassy S, Zeyaur KR, Ochatum N, Subramanian S. Maize-legume intercropping and pushpull for management of fall armyworm, stemborers, and striga in Uganda. Agron J. 2018;110:251322.

41. Kassie M, Bulte E, Balew S, Abro Z, Asale A. Social networks, incentives, and diffusion of house screening and push-pull technology interventions in rural Ethiopia. AEA RCT Registry. April 06. https://doi.org/10.1257/rct.5642-1.0. 2020.

42. Coetzee M. Key to the females of Afrotropical Anopheles mosquitoes (Diptera: Culicidae). Malar J. 2020;19:1-20. doi:10.1186/s12936-020-3144-9.

43. Beier, J. C., Perkins, P. V., Wirtz, R. A., Koros, J., Diggs, D., Gargan, T. P., \& Koech DK. Blood meal mosquito identificiation. J Med Entomol. 1988;25:9-16.

44. WHO. Haemoglobin concentrations for the diagnosis of anaemia and assessment of severity. 2011. doi:2011.

45. Kinde S, Ashine T, Massebo F, Woldeyes D. Acta Tropica Exploring the impact of house screening intervention on entomological indices and incidence of malaria in Arba Minch town, southwest Ethiopia: A randomized control trial. Acta Trop. 2018;181 October 2017:84-94. doi:10.1016/j.actatropica.2018.02.009.

46. WHO. Guidelines for monitoring the durability of long-lasting insecticidal mosquito nets under operational conditions. Geneva, Switzerland: World Health Organization; 2011.

47. FMOH. Diagnosis and Treatment Guidelines for Health Workers in Ethiopia 2nd Edition. Addis Ababa; 2004.

48. Killeen GF, Masalu JP, Chinula D, Fotakis EA, Kavishe DR, Malone D, et al. Control of Malaria Vector Mosquitoes by Insecticide-Treated Combinations of Window Screens and Eave Baffles. 2017;23.

49. WHO. Control of residual malaria parasite transmission. 2014;11 September:1-5.

50. Deressa W, Loha E, Balkew M, Hailu A, Gari T, Kenea O, et al. Combining long-lasting insecticidal nets and indoor residual spraying for malaria prevention in Ethiopia: Study protocol for a cluster randomized controlled trial. Trials. 2016;17:1-16. doi:10.1186/s13063-016-1154-2.

51. Kassie M, Abro Z, Wossen T, Ledermann ST, Diiro G, Ballo S, et al. Integrated Health Interventions for Improved Livelihoods: A Case Study in Ethiopia. 2020;:1-21.

52. WHO. Guidelines for Malaria Vector Control. Geneva, Switzerland: World Health Organization; 2019. http://www.ncbi.nIm.nih.gov/pubmed/30844152.

53. Hackett L. Malaria in Europe. An ecological study. Oxford: Oxford University Press; 1937. 
54. Boyd MF. The Influence of Obstacles Unconsciously Erected Against Anophelines (Housing and Screening) Upon the Incidence of Malaria. Am J Trop Med Hyg. 1926;6:157-60.

55. Ondiba IM, Oyieke FA, Ong'amo GO, Olumula MM, Nyamongo IK, Estambale BBA. Malaria vector abundance is associated with house structures in Baringo County, Kenya. PLoS One. 2018;13:1-12.

56. Rek JC, Alegana V, Arinaitwe E, Cameron E, Kamya MR, Katureebe A, et al. Articles Rapid improvements to rural Ugandan housing and their association with malaria from intense to reduced transmission: a cohort study. Lancet Planet Heal. 2018;2:e83-94. doi:10.1016/S25425196(18)30010-X.

57. Tusting LS, Rek J, Arinaitwe E, Staedke SG, Kamya MR, Cano J, et al. Why is malaria associated with poverty? Findings from a cohort study in rural Uganda. Infect Dis Poverty. 2016;:1-11. doi:10.1186/s40249-016-0164-3.

\section{Figures}

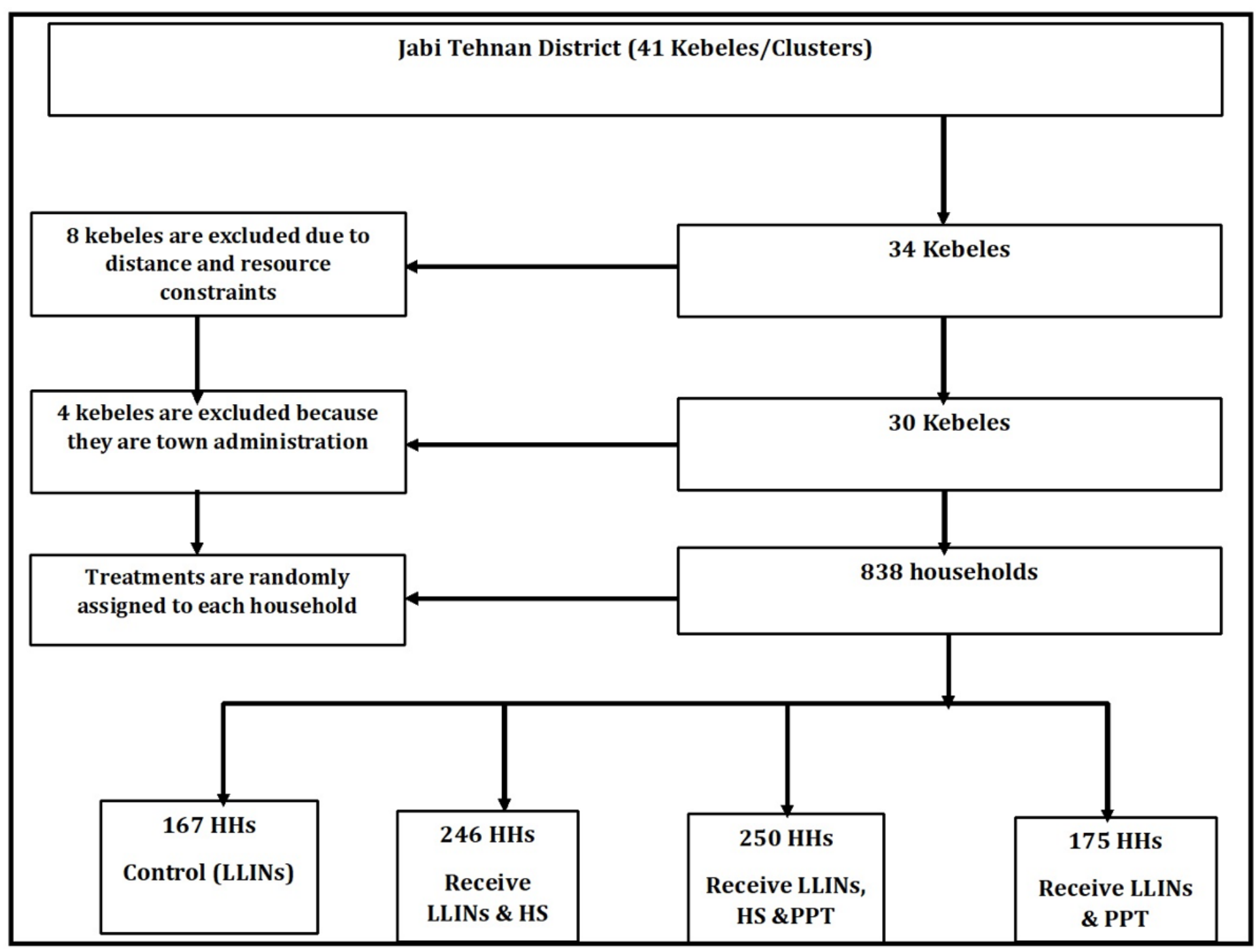

Figure 1 
Flow chart of the study

Page 24/24 